\title{
On the Graceful Game
}

\author{
Luisa Frickes $^{1}$, Simone Dantas ${ }^{1}$, Atílio G. Luiz ${ }^{3}$ \\ ${ }^{1}$ Instituto de Matemática e Estatística (IME) - Universidade Federal Fluminense (UFF) \\ Niterói - RJ - Brazil \\ ${ }^{2}$ Universidade Federal do Ceará (UFC) - Campus Quixadá \\ Quixadá, CE - Brazil \\ frickesluisalid.uff.br, sdantaseim.uff.br, gomes.atiliodufc.br
}

\begin{abstract}
A graceful labeling of a graph $G$ with $m$ edges consists in labeling the vertices of $G$ with distinct integers from 0 to $m$ such that, when each edge is assigned the absolute difference of the labels of its endpoints, all induced edge labels are distinct. Rosa established two well known conjectures: all trees are graceful (1966) and all triangular cacti are graceful (1988). In order to contribute to both conjectures we study these problems in the context of graph games. The graceful game was introduced by Tuza in 2017 as a two-players game on a connected graph in which the players Alice and Bob take turns labeling the vertices with distinct integers from 0 to $\mathrm{m}$. Alice's goal is to gracefully label the graph as Bob's goal is to prevent it from happening. In this work, we present the first results in this area by showing winning strategies for Alice and Bob in complete graphs, paths, cycles, complete bipartite graphs, caterpillars, prisms, wheels, helms, webs, gear graphs, hypercubes and some powers of paths.
\end{abstract}

\section{Introduction}

Graph labeling is an area of graph theory that has been attaining a particular importance since the 1960's. The main concern in this area consists in determining the feasibility of assigning labels to the elements of a graph satisfying certain conditions. Usually, the labels are elements of a set that supports some kind of mathematical operation.

One of the oldest and most studied graph labelings is the graceful labeling, so named by S. W. Golomb [Golomb 1972] and initially introduced by A. Rosa [Rosa 1967] around 1966. A graceful labeling of a graph $G=(V(G), E(G))$ with $m$ edges is an injective function $f: V(G) \rightarrow\{0,1, \ldots, m\}$ such that, when each edge $u v \in E(G)$ is assigned the (induced) label $g(u v)=|f(u)-f(v)|$, all induced edge labels are distinct. A graph $G$ that has a graceful labeling is called graceful.

Labeling problems are usually studied from the perpective of determining whether a given graph has a required labeling or not. An alternative outlook is to analyze labeling problems from the point of view of combinatorial games. The study of combinatorial games is a classical area in both discrete mathematics and game theory [Berlekamp et al. 1982]. One of the main characteristics of these types of games is that there is absolutely no lucky involved, that is, all players have perfect information and involves no chance. In most combinatorial games, two players - traditionally called Alice and Bob - alternately select and label vertices or edges (typically one vertex or edge in each step) in a graph $G$ which is completely known for both players. 
In a recent survey, Z. Tuza [Tuza 2017] proposes new labeling games, such as the graceful game studied in this work. Informally, the graceful game is a two-players game on a connected graph in which Alice and Bob take turns labeling the vertices of a graph $G$ with distinct numbers from the set $\{0,1, \ldots,|E(G)|\}$. Alice's goal is to gracefully label the graph as Bob's goal is to prevent it from happening.

In this work, we present the first results in the graceful game area and we contribute to the study of Rosa's conjectures: all trees are graceful [Rosa 1967] and all triangular cacti are graceful [Rosa 1988]. We study winning strategies for Alice and Bob in the following families of graphs: paths, cycles, complete bipartite graphs, complete graphs, caterpillars, hypercubes, helms, webs, gear graphs, prisms and 2-nd power of path. The results of this paper were developed by the student Luisa Frickes under the advisory of professors Simone Dantas and Atílio Luiz. They were presented at the 17th CologneTwente Workshop on Graphs \& Combinatorial Optimization University of Twente 2019 (CTW 2019) and published in its proceedings [Frickes et al. 2019].

This paper is organized as follows. Section 2 presents some definitions and auxiliary results used in our proofs. Section 3 presents our main results on the graceful game for some classic families of graphs. Finally, in Section 4 and Section 5, we present our conclusions and acknowledgements, respectively.

\section{Some definitions and auxiliary lemmas}

Given a simple graph $G$ and two different vertices $u, v \in V(G)$, the distance between $u$ and $v$ in $G$ is the number of edges in a shortest path between $u$ and $v$, and is denoted by $d_{G}(u, v)$. A graph's diameter is the maximum distance between any pair of vertices in $G$. The $k$-th power of a simple graph $G$ is the simple graph $G^{k}$ that has $V\left(G^{k}\right)=V(G)$, with distinct vertices $u, v$ being adjacent in $G^{k}$ if and only if $d_{G}(u, v) \leq k$.

We say that two simple graphs $G$ and $H$ are $i$ somorphic, if there exists a bijection $\theta: V(G) \rightarrow V(H)$ such that any two vertices $u, v \in V(G)$ are adjacent in $G$ if and only if $\theta(u)$ and $\theta(v)$ are adjacent in $H$.

Given a graph $G$ with $m$ edges and with a graceful labeling $f$, the complementary labeling of $f$ is the labeling $\bar{f}$ defined as $\bar{f}(v)=m-f(v)$ for all $v \in V(G)$. The complementary labeling of a graceful labeling of $G$ is also graceful. An $\alpha$-labeling of a graph $G$ on $m$ edges is a graceful labeling $g$ with the additional property that, for every edge $u v \in E(G)$, either $g(u) \leq k<g(v)$ or $g(v) \leq k<g(u)$, for some integer $k \in\{0, \ldots, m\}$.

The graceful game is defined in the following way: Alice and Bob alternately assign an unused label $f(v) \in\{0, \ldots, m\}$ to a previously unlabeled vertex $v$ of a given simple graph $G=(V(G), E(G))$ with $m$ edges. We call a vertex of $G$ free if it is not labeled yet. If both endpoints of an edge $u v \in E(G)$ are already labeled, then the label of the edge $u v$ is defined as $|f(u)-f(v)|$. A move (label assignment) is said to be legal if, after it, all edge labels are distinct. In the graceful game, Alice wins if the whole graph $G$ is gracefully labeled, and Bob wins if he can prevent this.

It is well known that not every graph is graceful; in fact, most graphs are not graceful [Golomb 1972]. For non-graceful graphs, it is immediate that Bob is the winner and, therefore, the game is completely determined for such graphs. In this work, we 
investigate classes of graphs for which it is possible to obtain a graceful labeling. The next two lemmas show properties of the graceful game that are used throughout this work.

Lemma 1. Let $G$ be a simple graph with $m$ edges. Alice can only use the label 0 (resp. $m$ ) to label a vertex $v \in V(G)$ if $v$ is adjacent to every remaining free vertex or $v$ is adjacent to a vertex already labeled by Bob with $m$ (resp. 0).

Lemma 2. Let $G$ be a simple graph with $m$ edges. If Bob assigns label 0 (resp. $m$ ) to a vertex $v \in V(G)$, such that $v$ has only one free neighbor or there are two free vertices in $G$ not adjacent to $v$, then Alice is forced to label a vertex adjacent to $v$ with $m$ (resp. 0 ).

\section{Main results}

In this section we present the results on each class of graphs approached in the research.

A path graph $P_{n}$ is a connected graph on $n$ vertices whose vertices can be arranged in a linear sequence $\left(v_{0}, v_{1}, \ldots, v_{n-1}\right)$ in such a way that two vertices are adjacent if and only if they are consecutive in the linear sequence. Rosa [Rosa 1967] proved that all paths are graceful.

Theorem 3. Bob has a winning strategy for any $P_{n}, n \geq 4$. For $n=3$ the winner is the player who starts the game, and Alice has a winning strategy for $n \in\{1,2\}$.

Sketch of the proof. It is trivial that Alice always wins on $P_{1}$ and $P_{2}$. For $P_{3}$, if Bob starts, it is sufficient that he labels $v_{1}$ with 1 , so that Alice cannot get the edge label 2. In contrast, if Alice starts, she labels $v_{0}$ with 1 , and independently of Bob's choice, the graph is graceful. For $P_{n}, n \geq 4$, we show that Bob can exhaust Alice's possibilities of creating the edge label $m-1$ when he is the first player. Now, when Alice is the first one to play, paths $P_{4}$ and $P_{n}, n \geq 5$, are treated separately. For the first one, we refer to the only two graceful labelings of $P_{4}$, and, considering Lemma 1, we show that there is no move Alice can make that leads her to the victory. For the second one, we show that Bob can always exhaust Alice's possibilities of creating edge label $m$ or $m-1$.

A complete graph $K_{n}$ is a simple graph in which every pair of distinct vertices is connected by one edge. Golomb [Golomb 1972] proved that $K_{n}$ is graceful if and only if $n \leq 4$.

Theorem 4. Alice wins on $K_{3}$ and Bob on $K_{4}$, no matter who starts.

Sketch of the proof. The cases where $n=1$ or $n=2$ resemble to $P_{1}$ and $P_{2}$, thus, the results follow from Theorem 3. Graph $K_{3}$ is graceful if and only if two of its vertices are assigned labels 0 and 3. Therefore, Alice's strategy, when she is the first player, is to assign these labels to vertices of the graph. On the other hand, when Bob is the first player he must begin by labeling a vertex with 1 or 2 . In both cases, Alice can induce an illegal move by Bob if he tries to complete his goal. This way, his only possibility is to label the last vertex in Alice's favor. Regarding to $K_{4}$, we refer to its only two graceful labelings to conclude that none of it assigns 3 to its vertices. Hence, no matter who starts, Bob can label a vertex with 3 , winning the game. 
A cycle graph $C_{n}$, with $n \geq 3$ vertices, is a connected simple graph such that all of its vertices can be arranged in a cyclic sequence $\left(v_{0}, v_{1}, \ldots, v_{n-1}\right)$ such that two vertices are adjacent if and only if they are consecutive in the sequence. Rosa [Rosa 1967] proved that the cycle graph $C_{n}$ is graceful if and only if $n \equiv 0,3 \quad(\bmod 4)$.

The proof of Theorem 5 follows Theorem 4 for $C_{3} \cong K_{3}$, and uses similar strategy for $C_{4}$, guided by its only two graceful labelings. Generalizing to $C_{n}, n>4$ and $n \equiv 0,3$ $(\bmod 4)$, Bob can exhaust Alice's chances of creating the edge label $n-1$.

Theorem 5. Bob has a winning strategy for $C_{n}, n \geq 4$, and Alice wins on $C_{3}$.

A bipartite graph is a graph $G=(V(G), E(G))$ such that there exists a partition $P=(X, Y)$ of $V(G)$ such that, for every edge $u v \in E(G), u \in X$ and $v \in Y$. A complete bipartite graph $K_{p, q}$ is a bipartite graph in which each vertex of $X$ is joined to every vertex of $Y$, with $p=|X|$ and $q=|Y|$. Note that $\left|V\left(K_{p, q}\right)\right|=p+q$ and $\left|E\left(K_{p, q}\right)\right|=p q$.

Theorem 6. Bob has a winning strategy for all $K_{p, q}, p, q \geq 2$. Alice wins the Graceful game in any star $K_{1, q}$ if she is the first player.

Sketch of the proof. Results for $K_{1,0}, K_{1,1}$ and $K_{1,2}$ follows from Theorem 3. For $K_{1, q}$, $q \geq 3$, we consider $P=(X, Y)$ a bipartition of $K_{1, q}$ and $|X|=1$ (wlog). Alice's strategy when she is the first player is to label the vertex in $X$ with 0 ou $q$. In the other hand, when Bob is the first player, it suffices him to label this same vertex with any label but 0 ou $q$. The result for $K_{2,2} \cong C_{4}$ follows from Theorem 5. Consider $K_{p, q}, p \geq 2$ and $q \geq 3$, and let $(X, Y)$ be a bipartition of $K_{p, q}$. When Bob is the first player he establishes a pattern of moves that forces Alice to assign specific labels to vertices in $Y$ till all of its free vertices are exhausted. When $q$ is even, Bob can prevent Alice from creating the edge label $m-\frac{q}{2}$. When $q$ is odd, in order to get the edge label $m-\left(\frac{q}{2}+1\right)$, Alice would create a second edge label $\frac{q}{2}$, making it impossible for her to do so.

When Alice is the first player, we address $K_{2, q}, q \geq 3$, and $K_{p, q}, p \geq 3$ and $q \geq 3$, separately. For $K_{2, q}, q \geq 3$, we show that Bob can prevent Alice from creating the edge label $m-1$, since it would generate a repeated edge label $i-1$ or $m-i-1, i$ being the label she chooses for her first move. The case $K_{p, q}, p \geq 3$ and $q \geq 3$, is divided in the following way. Case 1: $x<i<m-x$, where $x=\left(\frac{p-2}{2}\right)$ if $p$ is even; or $x=\left(\frac{p-1}{2}\right)$, otherwise. If $p$ is odd, it takes place the same pattern that occurs when Bob is the first player, until the free vertices in $X$ are exhausted. If $p$ is even, we show Bob can induce Alice into a move that creates a repeated edge label $x=|(x+1)-1|$. Therefore, Alice looses the game. Case 2: $i=k$ or $i=m-k$, for $1 \leq k \leq x$, where $x=\left(\frac{p-2}{2}\right)$ if $p$ is even; or $x=\left(\frac{p-1}{2}\right)$, otherwise. If $i=k(i=m-k$ is analogous), Bob can prevent Alice from creating the edge label $m-k$.

A caterpillar cat $\left(k_{1}, k_{2}, \ldots, k_{s}\right)$ is a special tree obtained from a path $P=$ $\left(v_{1}, v_{2}, \ldots, v_{s}\right)$, called spine, by joining $k_{j}$ leaf vertices to $v_{j}$, for each $j \in\{1, \ldots, s\}$. Rosa [Rosa 1967] proved that every caterpillar has an $\alpha$-labeling.

If a caterpillar $H$ has diameter at most two, then $H$ is isomorphic to a star $K_{1, m}$ and, we apply Theorem 6 . Theorem 8 characterizes the graceful game for caterpillars with diameter at least three. Lemma 7 is used in the proof of Theorem 8. 
Lemma 7. Let $H=\operatorname{cat}\left(k_{1}, k_{2}, \ldots, k_{s}\right)$ be a caterpillar with $m$ edges and $v_{j}$ be an arbitrary vertex in the spine with $k_{j}>0$ adjacent leaves, for $j \in\{1, \ldots, s\}$. If there exists a leaf $u$ not yet labeled adjacent to $v_{j}$ and, the colors 0 or $m$ have not been used, then Alice cannot label $v_{j}$ with any color.

Theorem 8. Bob has a winning strategy for all caterpillars with diameter at least three.

Sketch of the proof. The strategy taken by Bob when he is the first player is to prevent Alice from creating the edge label $m-1$ by labeling a leaf $u$ with 0 , then, using label 1 on a vertex not adjacent $u$ 's "spine vertex". When Alice is the first player, by Lemma 7, she can only label a leaf or a vertex $v_{j}$ in the spine whose $k_{j}=0, j \in\{1, \ldots, s\}$. In both cases, Bob can also prevent her from creating the edge label $m-1$ by similar strategy. Although, the previous strategy cannot be applied to $H=\operatorname{cat}\left(1, k_{2}\right)$. Thus, this graph is approached differently and the proof depends on the parity of $m$. For either $m$ even or $m$ odd, Bob can manage a sequence of moves in which Alice, trying to create all the edge labels for a graceful labeling, is faced with a move in which she would generate a repeated edge label $i-1$ and $i$, respectively.

A wheel $W_{n}$ is a graph formed by connecting a single vertex $v_{n}$, called central vertex of $W_{n}$, to all vertices $v_{0}, v_{1}, \ldots, v_{n-1}$ of a cycle $C_{n}$, where $n \geq 3$. By definition, a wheel $W_{n}$ has $n+1$ vertices and $2 n$ edges. In 1987, C. Hoede and H. Kuiper [Hoede and Kuiper 1987] proved that all wheels are graceful. Lemma 9 immediately implies Theorem 10.

Lemma 9 ([Frucht 1979]). Let $v_{n}$ be the central vertex of the wheel graph $W_{n}$. There exist no graceful labeling $f$ of $W_{n}$ with $f\left(v_{n}\right)=n$.

Theorem 10. When Bob is the first player, he has a winning strategy for all wheel graphs.

Theorem 11. Bob has a winning strategy for the wheel graphs $W_{3}, W_{4}$ and $W_{5}$ even when Alice is the first player.

Sketch of the proof. For $n=3$, the result follows from Theorem 4 since $W_{3} \cong K_{4}$. For $n=4$, we refer to the eight possible graceful labelings of $W_{4}$, obtained through computational search. In that case, we reached only two options for Alice: either (a) she labels a vertex other than $v_{4}$ with 4 (precluding Bob from assigning label 4 to the central vertex); or (b) she labels $v_{4}$ with a label $i \in\{0,1, \ldots, 8\}$ and $i \neq 4$ (by Lemma 1 and Lemma 9). In (a), she is forced by Bob to make a set of moves that leads to labelings of $W_{4}$ that are not graceful. In (b), no matter what move Alice makes, Bob always has a label he can use on his next move in which precludes Alice from gracefully labeling the graph.

For $W_{5}$, the case where Alice labels a vertex $v_{j}, 0 \leq j \leq, 4$, and the one she labels $v_{5}$ are treated separately. In the first one, Bob makes a set of moves that leads Alice into a move that creates a second edge label 1 . The second case is divided into four subcases. In Subcases 2.1 and 2.2 (Alice labels $v_{5}$ with a label $i, 3 \leq i \leq 8$ and $i \neq 5$, and, Alice labels $v_{5}$ with $i=2$, respectively), Bob makes it impossible for Alice to create the edge label 9. In Subcase 2.3 (Alice labels $v_{5}$ with $i \in\{1,9\}$ ), Bob forces Alice into a move that would 
generate a second edge label 1. In Subcase 2.4 (Alice labels $v_{5}$ with $i=0$ (resp. $i=10$ )), we refer to all four graceful labelings of $W_{5}$ that assign label 0 (resp. $i=10$ ) to $v_{5}$ in order to conclude that Bob can prevent Alice from gracefully labeling the graph.

The approach used in the proof of Theorem 11 for $W_{5}$ can almost be successfully extended for arbitrary wheels $W_{n}$ with $n \geq 6$, with exception of Subcase 2.4. However, we conjecture that Bob has a winning strategy for all these graphs.

Conjecture 12. Bob has a winning strategy for all wheel graphs $W_{n}$ with $n \geq 6$.

A gear graph $G_{n}, n \geq 3$, is a simple graph obtained by subdividing each edge of the outer $n$-cycle $\left(v_{0}, v_{1}, \ldots, v_{n-1}\right)$ of a wheel graph $W_{n}$ exactly once. The vertices of $G_{n}$ are named as follows: $v_{n} \in V\left(G_{n}\right)$ is the original central vertex of $W_{n}$, $v_{0}, v_{1}, \ldots, v_{n-1}$ are the original vertices of the outer $n$-cycle (these vertices are adjacent to $v_{n}$ ) and, for each $j \in\{0, \ldots, n-1\}, w_{j}$ is the vertex adjacent to $v_{j}$ and $v_{j+1}$, indices taken modulo $n$. Note that, by definition, $G_{n}$ has $2 n+1$ vertices and $3 n$ edges. Ma and Feng [Ma and Feng 1984] proved in 1984 that all gear graphs are graceful.

The strategies taken to show, in Theorem 13, that Bob wins in all gear graphs are set as follows. When Bob is the first player, it suffices him to label a vertex $w_{j}$ with 0 in order to preclude Alice from creating the edge label $m-1$. When we address Alice as the first player we divide the proof into three different cases: (i) she starts labeling a vertex $w_{j}, 0 \leq j \leq n-1$; (ii) she starts labeling a vertex $v_{j}, 0 \leq j \leq n-1$; (iii) she starts labeling $v_{n}$. In all of them, Bob precludes Alice from creating the edge label $m-1$. The graph $G_{3}$ has particularities and it is, therefore, treated separately using its graceful labelings. We obtain that Alice cannot start the game by labeling $v_{3}$ since Bob wins by choosing between 0 and 9 to label a neighbor of $v_{3}$. In addition, if Alice starts by labeling a vertex other than $v_{3}$, Bob will also prevent her from gracefully labeling the graph.

Theorem 13. Bob has a winning strategy for all gear graphs.

A helm $H_{n}, n \geq 3$, is the graph obtained from $W_{n}$ creating a new vertex for each non-central vertex $v_{j}$ of $W_{n}$, and linking $v_{j}$ with this new vertex. By definition, a helm $H_{n}$ is a graph on $3 n$ edges and $2 n+1$ vertices, that are named as follows: the unique vertex with degree $n$ is called center and is denoted by $v_{0}$; the neighbors of $v_{0}$ are called cycle vertices and are denoted by $v_{1}, \ldots, v_{n}$; the remaining vertices are called pendent vertices and are denoted by $v_{n+1}, \ldots, v_{2 n}$. Moreover, we consider that $v_{k}$ and $v_{n+k}, 1 \leq k \leq n$, are adjacent and are arranged linearly. In 1984, Ayel [Ayel and Favaron 1984] proved that all helms are graceful.

Lemma 14. Given a helm $H_{n}$, for $j \in\{1, \ldots, n\}$, Alice can label a cycle vertex $v_{j}$ of $H_{n}$ in only two cases: when $v_{j}$ 's respective pendent vertex is already labeled or, when the colors 0 or $3 n$ (or both) have already been assigned to a vertex.

Theorem 15. Bob has a winning strategy for all helms.

Proof. Given a helm graph $H_{n}, n \geq 3$, consider the case where Bob is the first player. He starts by labeling a pendent vertex $v_{n+j}, 1 \leq j \leq n$, with 0 . According to Lemma 2, Alice is forced to label $v_{j}$ with $3 n$. Then, Bob assigns label 1 to a vertex not adjacent to $v_{j}$. Since there are no possibilities left for Alice to create the edge label $3 n-1$, Bob wins 
the game. Now, suppose that Alice is the first player. By Lemma 14, she has only two options for her first move: to label the center or a pendent vertex. Suppose Alice chooses to label the center with a arbitrary color $i, 1 \leq i \leq 3 n-1$. If $i=1$ (resp. $i \neq 1$ ), then Bob labels a pendent vertex $v_{n+p}, 1 \leq p \leq n$, with $3 n$ (resp. 0 ). Alice is now forced to label $v_{p}$ with 0 (resp. $3 n$ ). In order to win, Bob labels a vertex not adjacent to $v_{p}$ with $3 n-1$ (resp. 1). Next, suppose that Alice chooses to label a pendent vertex $v_{n+j}, 1 \leq j \leq n$, with an arbitrary color $i, 1 \leq i \leq 3 n-1$. Then, Bob assigns 0 to a second pendent vertex $v_{n+p}, 1 \leq p \leq n$ and $p \neq j$. This way, Alice is forced to label $v_{p}$ with $3 n$. If $i=1$, then Bob wins since there is no way for Alice to generate the edge label $3 n-1$. If $i \neq 1$, Bob assigns label 1 to a vertex not adjacent to $v_{p}$ and the result follows.

Web graphs were defined by Koh et al. [Kho et al. 1980] as a graph obtained by connecting the pendent vertices $v_{n+1}, v_{n+2}, \ldots, v_{2 n}$ of a helm into a cycle $\left(v_{n+1}, v_{n+2}, \ldots, v_{2 n}\right)$ and then linking a single new pendent vertex to each vertex of this outer cycle. Later, Kang et al. [Kang et al. 1996] extended the definition of web graphs so that the process of creating a new cycle by joining the pendent vertices and adding a pendent edge to each vertex of the outer cycle could be repeated as many times as desired. In this paper, we use the definition proposed by Kang et al. [Kang et al. 1996], in which $W(t, n)$ denotes the web graph formed by $t$ vertex-disjoint $n$-cycles, where $t \geq 2$. These $t n$-cycles are called the concentric cycles of $W(t, n)$.

By definition, a web graph $W(t, n)$ has $n(t+1)+1$ vertices and $m=n(2 t+1)$ edges. We partition the vertex set of $W(t, n)$ into $t+2$ parts. The first part comprises only the central vertex $v_{0}$, also called center. The second part comprises the pendent vertices, denoted by $v_{1}, v_{2}, \ldots, v_{n}$. The other $t$ parts are each one formed by the vertices that give rise to each concentric cycle. We denote $v_{n+1}, v_{n+2}, \ldots, v_{2 n}$ the vertices of the outer concentric cycle; $v_{2 n+1}, v_{2 n+2}, \ldots, v_{3 n}$ the vertices of the next concentric cycle, and so on, until the inner concentric cycle $v_{t n+1}, v_{t n+2}, \ldots, v_{(t+1) n}$. We also consider that $v_{k}, v_{n+k}, v_{2 n+k}, \ldots, v_{t n+k}, v_{0}$, for any $k \in\{1, \ldots, n\}$, are arranged linearly.

Kang et al. [Kang et al. 1996] proved that $W(2, n), W(3, n), W(4, n)$ are graceful and, Abhyankar [Abhyankar 2002] proved the same for $W(t, 5), 5 \leq t \leq 13$. Even though there are not many results for the gracefulness of the web graphs, we provide a strategy in which we guarantee that Bob is the winner on any web graph.

Lemma 16. Given a web graph $W(t, n)$ with $m$ edges, Alice can label an outer cycle vertex $v_{j}, j \in\{n+1, \ldots, 2 n\}$, in only two cases: when $v_{j}$ 's respective pendent vertex is already labeled or, when the colors 0 or $m$ (or both) have already been assigned to a vertex.

Theorem 17. Bob has a winning strategy for all webs.

Proof. Let $W(t, n)$ be a web graph with $m$ edges. When Bob is the first player he assigns label 0 to a pendent vertex $v_{j}, j \in\{1, \ldots, n\}$. By Lemma 2, Alice's only option is to label $v_{n+j}$ with $m$. Now, Bob assigns label 1 to a vertex not adjacent to $v_{n+j}$ and he wins. Now, suppose that Alice is the first player. By Lemma 16 and by the symmetry of the graph, we conclude that Alice has only three options for her first move. Option 1: Alice labels $v_{0}$ with an arbitrary color $i \in\{1, \ldots, m-1\}$. In this case, Bob assigns the color 0 to a pendent vertex $v_{p}, p \in\{1, \ldots, n\}$, forcing Alice to label $v_{n+p}$ with $m$. If $i=1$, then 
the game is over and Bob is the winner since there is no possibility left for Alice to create the edge label $m-1$. If $i \neq 1$, in order to win the game Bob labels any vertex that is not adjacent to $v_{n+p}$ with 1. Option 2: Alice labels a pendent vertex $v_{j}, j \in\{1, \ldots, n\}$ with an arbitrary color $i \in\{1, \ldots, m-1\}$. In this case, Bob labels a second pendent vertex $v_{p}$, $p \in\{1, \ldots, n\}$ and $p \neq j$, with 0 . Alice is now forced to label $v_{n+p}$ with $m$. If $i=1$ then the game is over and Bob wins. If $i \neq 1$, Bob labels any vertex that is not adjacent to $v_{n+p}$ with 1 in order to win. Option 3: Alice labels a vertex $v_{j}, j \in\{2 n+1, \ldots,(t+1) n\}$, with an arbitrary color $i \in\{1, \ldots, m-1\}$. In this case, Bob assigns label 0 to a pendent vertex $v_{p}, p \in\{1, \ldots, n\}$, so that $v_{j}$ and $v_{p}$ are not arranged linearly. This forces Alice to label $v_{n+p}$ with $m$. If $i=1$ then the game is over and Bob wins. If $i \neq 1$, Bob must label any vertex that is not adjacent to $v_{n+p}$ with 1 and he also wins.

An $n$-dimensional hypercube $Q_{n}$ is defined recursively in terms of the Cartesian product of two graphs as follows: (i) $Q_{1}=K_{2}$; and (ii) $Q_{n}=K_{2} \square Q_{n-1}$. It is well known [Harary et al. 1988] that every $Q_{n}$ is bipartite, $n$-regular and has $\left|V\left(Q_{n}\right)\right|=2^{n}$ and $\left|E\left(Q_{n}\right)\right|=n 2^{n-1}$. In 1981, Kotzig [Kotzig 1981] proved that all hypercubes are graceful.

The strategies used to prove Theorem 18 follow the ideas presented next. Since $Q_{2} \cong C_{4}$, its result follows from Theorem 5. For $Q_{n}, n \geq 3$, the reasoning used when Bob is the first player is similar to the one used for the complete bipartite when he is also the first one to play. As for when Alice is the first player, she starts by labeling $v_{1} \in X$ with an arbitrary label $i$ and we split into four different cases: (i) $i=1$ or $i=m-1$; (ii) $2 \leq i \leq\left\lfloor\frac{n}{2}\right\rfloor$; (iii) $\left\lfloor\frac{n}{2}\right\rfloor<i<m-\left\lfloor\frac{n}{2}\right\rfloor$; and (iv) $i=m-k$ for $2 \leq k \leq\left\lfloor\frac{n}{2}\right\rfloor$. We show Bob can prevent her from creating the edge labels $m-1, m-i, m-\lfloor n / 2\rfloor$ and $m-k$ on each case, respectively.

Theorem 18. Bob has a winning strategy for all hypercubes $Q_{n}$ with $n \geq 2$.

The prism graph $P_{r, 2}, r \geq 3$, is defined as the cartesian product $C_{r} \square P_{2}$ of a cycle on $r$ vertices and path $P_{2}$. Frucht and Gallian [Frucht and Gallian 1988] proved that every prism is graceful.

In the proof of Theorem 19, we approach $P_{r, 2}, r \geq 4$ and $P_{3,2}$ separately. For $P_{r, 2}, r \geq 4$, when Bob is the first player, we show he can prevent Alice from creating the edge label $m-2$ and, when Alice is the first one to play, we divide it into Case 1, $2 \leq i \leq m-2$, and, Case 2, $i \in\{1, m-1\}$, where, in both cases, $i$ is the color assigned by Alice on her first move. In both cases, Bob can prevent Alice from creating the edge label $m-1$, therefore, winning the game.

Theorem 19. Bob has a winning strategy for all prisms.

The family of powers of paths comprises all graphs $G^{k}$ obtained when $G \cong P_{n}$, $n \geq 1$. The $k$-th power of a path $P_{n}$ is denoted by $P_{n}^{k}$. It is known that all graphs $P_{n}^{2}$ are graceful [Kang et al. 1996] and Theorem 20 characterizes the graceful game for $P_{n}^{2}$.

Theorem 20. Bob has a winning strategy for all $P_{n}^{2}$ with $n \geq 4$. Alice wins on $P_{3}^{2}$.

Sketch of the proof. Since $P_{1}^{2} \cong P_{1}, P_{2}^{2} \cong P_{2}$ and $P_{3}^{2} \cong C_{3}$, the result for these graphs follows from Theorems 3 and 5. Now, consider $P_{n}^{2}, n \geq 3$. When Bob is the first player, 
he assigns 0 to $v_{0}$, forcing Alice to assign $m$ to $v_{1}$ or $v_{2}$ (Lemma 2). Regardless of her option, Bob can prevent her from creating the edge label $m-1$ (using the rule of the game in his favor) and, the edge label $m-2$, respectively. The instance where Alice is the first player is divided into seven cases. Case 1: $P_{n}^{2}, n \geq 10$. Bob can induce Alice into a move that, in order to create edge label $m-2$, she would generate a repeated edge label $\frac{m-3}{2}$. Case 2: $P_{4}^{2}$. We refer to all graceful labelings of $P_{4}^{2}$ to show that, no matter what Alice's move, Bob can always preclude her from gracefully labeling the graph. Case 3: $P_{5}^{2}$. Subdivided into eight cases. In all subcases, Bob induces Alice into a illegal move, precluding her from achieving her goal. Case 4: $P_{6}^{2}$. Subdivided into four cases. In Subcases 4.1 to 4.4, Bob prevents her from creating the edge label 8. Subcase 4.5 applies to Case 1. Case 5: $P_{7}^{2}$. Case 6: $P_{8}^{2}$. Case 7: $P_{9}^{2}$. All three last cases applies to Cases 1 and 4.

\section{Conclusion}

The results of this work are the first in the area of graceful labeling games, opened in 2017 by Tuza [Tuza 2017], and they also contribute to the study of the two famous graceful graph conjectures posed by Rosa [Rosa 1967, Rosa 1988]. We note that the techniques used in the proofs have a common core but they are strongly dependent on the graph structure, the player who initiates, the order of the chosen labels, and therefore it is not possible to apply a generalization for all classes.

Table 1. Graph classes and winners: A (Alice) and B (Bob).

\begin{tabular}{ccc}
\hline \multirow{2}{*}{ Graph class } & \multicolumn{2}{c}{ First player } \\
\cline { 2 - 3 } & Alice & Bob \\
\hline$P_{n}, n=1,2$ & $\mathrm{~A}$ & $\mathrm{~A}$ \\
\hline$P_{3}$ & $\mathrm{~A}$ & $\mathrm{~B}$ \\
\hline$P_{n}, n \geq 4$ & $\mathrm{~B}$ & $\mathrm{~B}$ \\
\hline$K_{3}$ & $\mathrm{~A}$ & $\mathrm{~A}$ \\
\hline$K_{4}$ & $\mathrm{~B}$ & $\mathrm{~B}$ \\
\hline$C_{n}, n \geq 4$ & $\mathrm{~B}$ & $\mathrm{~B}$ \\
\hline$K_{1, q}, q \geq 2$ & $\mathrm{~A}$ & $\mathrm{~B}$ \\
\hline$K_{p, q}, p, q \geq 2$ & $\mathrm{~B}$ & $\mathrm{~B}$ \\
\hline$c a t\left(k_{1}, \ldots, k_{s}\right), s \geq 2$ & $\mathrm{~B}$ & $\mathrm{~B}$ \\
\hline$W_{n}, n=3,4,5$ & $\mathrm{~B}$ & $\mathrm{~B}$ \\
\hline$W_{n}, n \geq 6$ & $?$ & $\mathrm{~B}$ \\
\hline$H_{n}, n \geq 3$ & $\mathrm{~B}$ & $\mathrm{~B}$ \\
\hline$W(t, n), t \geq 2, n \geq 3$ & $\mathrm{~B}$ & $\mathrm{~B}$ \\
\hline$G_{n}, n \geq 3$ & $\mathrm{~B}$ & $\mathrm{~B}$ \\
\hline$Q_{n}, n \geq 2$ & $\mathrm{~B}$ & $\mathrm{~B}$ \\
\hline$P_{n}^{2}, n \geq 4$ & $\mathrm{~B}$ & $\mathrm{~B}$ \\
\hline
\end{tabular}

Our results are summarized in Table 1 . We observe that Alice has winning strategies for only few cases such as: complete graphs $K_{i}, i \leq 3$, and stars $K_{1, q}, q \geq 2$ when she is the first player. We also leave it open the Conjecture 12, in which we believe that 
Bob has a winning strategy for all $W_{n}, n \geq 6$. This conjecture also contributes to the continuity of studies in the area.

\section{Acknowledgements}

This study was financed in part by the Coordenação de Aperfeiçoamento de Pessoal de Nível Superior - Brasil (CAPES) - Finance Code 001, CAPES-PrInt project number 88881.310248/2018-01, CNPq and FAPERJ (CNE E26/200.895/2018, IC E26/200.323/2019).

\section{References}

Abhyankar, V. J. (2002). Direct Methods of Gracefully Labeling Graphs. Ph.d. thesis, University of Mumbai.

Ayel, J. and Favaron, O. (1984). Helms are graceful. in Progress in Graph Theory (Waterloo, Ont., 1982), Academic Press, Toronto, Ont., pages 89-92.

Berlekamp, E. R., Conway, J. H., and Guy, R. K. (1982). Winning ways for your mathematical plays. Academic Press.

Frickes, L., Dantas, S., and Luiz, A. G. (2019). The graceful game. In Proceedings of 17th Cologne-Twente Workshop on Graphs and Combinatorial Optimization (CTW 2019), volume 1, pages 41-44.

Frucht, R. (1979). Graceful numbering of wheels and related graphs. Annals of the New York Academy of Sciences, 319(1):219-229.

Frucht, R. and Gallian, J. A. (1988). Labeling prisms. Ars Combinatoria, 26:69-82.

Golomb, S. W. (1972). How to number a graph. In Read, R. C., editor, Graph Theory and Computing, pages 23-37. Academic Press, New York.

Harary, F., Hayes, J. P., and Wu, H.-J. (1988). A survey of the theory of hypercube graphs. Computers \& Mathematics with Applications, 15(4):277-289.

Hoede, C. and Kuiper, H. (1987). All wheels are graceful. Utilitas Mathematica, 14:311.

Kang, Q., Liang, Z.-H., Gao, Y.-Z., and Yang, G.-H. (1996). On labeling of some graphs. Journal of Combinatorial Mathematics and Combinatorial Computing, 22:193-210.

Kho, K. M., Rogers, D. G., Teo, H. K., and Yap, K. Y. (1980). Graceful graphs: some further results and problems. Congressus Numerantium, 29:559-571.

Kotzig, A. (1981). Decompositions of complete graphs into isomorphic cubes. Journal of Combinatorial Theory, B 31:292-2496.

Ma, K. J. and Feng, C. J. (1984). On the gracefulness of gear graphs. Mathematics in Practice and Theory, pages 72-73.

Rosa, A. (1967). On certain valuations of the vertices of a graph. Theory of Graphs, International Symposium, Rome, July 1966, pages 349-355.

Rosa, A. (1988). Cyclic steiner triple systems and labelings of triangular cacti. Scientia, 1:87-95.

Tuza, Z. (2017). Graph labeling games. Electronic Notes in Discrete Mathematics, 60:6168. 